\title{
Renal Replacement Lipomatosis
}

\author{
Y.Xu R.L. Liu Z.H. Zhang W.M. Zhao Q.C. Yang \\ Department of Urology, Second Hospital, and Tianjin Institute of Urology, Tianjin Medical University, Tianjin, China
}

\section{Key Words}

Renal replacement lipomatosis - Lipomatosis • Xanthogranuloma

\begin{abstract}
We report a relatively rare case of renal replacement lipomatosis presenting as a renal mass. Computed tomography revealed a predominantly low-density and roundish mass, with an irregular renal parenchyma, high-density calcification, and abundant low-density fat. The differential diagnosis before surgery was squamous cell carcinoma, teratoma, or angiomyolipoma of the kidney. The case was initially misdiagnosed, because we had no experience with this disease. After mass exploration, histological examination confirmed the diagnosis of renal replacement lipomatosis. The patient was free from signs of recurrence 10 months after the operation.

Copyright $\odot 2006$ S. Karger AG, Basel
\end{abstract}

\section{Introduction}

Renal replacement lipomatosis is a relatively rare benign disease of the kidney and represents a condition of proliferated fat within renal sinus, hilum, and perirenal space, replacing the renal parenchyma.

\section{Case Report}

A 62-year-old woman was admitted to our hospital, complaining of a mass in her right abdomen for 4 years. The patient noted the mass accidentally about 4 years ago, growing during these 4 years, and she claimed an 8-kg weight loss. The patient had a 20 -year history of bilateral renal stones and had undergone lithotomy once on the left side 20 years previously; the right kidney was not treated. On physical examination, a slightly tender and hard mass was palpable from the right upper abdomen. An abdominal radiograph demonstrated multiple right renal calculi. Intravenous urography showed a nonfunctioning right kidney and a nearly normal left kidney. A computed tomography (CT) scan revealed a predominantly low-density and roundish mass with an irregular renal parenchyma, high-density calcification, and abundant low-density fat (fig. 1). Magnetic resonance imaging indicated nearly the same impression as CT. Preoperative urinalysis showed pyuria, but no bacterial growth was observed. We were not able to make a definitive diagnosis preoperatively. We thought about the possibility of a teratoma or an angiomyolipoma of the kidney, but could not reach a final diagnosis. So, before the exploring operation, a consultation was held within our department, and this case was discussed. The differential diagnosis before the operation included squamous cell carcinoma (SCC), teratoma, or angiomyolipoma of the kidney, considering the long-time stone-stimulating and disease history. During mass exploration, right nephrectomy with perirenal fat resection was performed. Macroscopic findings were a $20 \times 15 \times 10$-cm solid mass, multiple calculi with extremely atrophied renal parenchyma surrounded by overgrown renal sinus, and perirenal fat. The whole kidney was almost replaced by fatty tissues (fig. 2). Microscopic examination indicated that the hyperplastic fatty tissue at renal sinus, hilum, and periphery was mature fat cells with infiltration of inflammatory cells (fig. 3). The renal structure was completely destroyed, and all glomeruli were hyaline with infiltration of a large amount of inflammatory cells (fig. 4). The postoperative diagnosis was renal replacement lipomatosis, and malignant conditions were excluded. At 10 months of follow-up, the patient was free from signs of recurrence.

\section{KARGER \\ Fax +41613061234 E-Mail karger@karger.ch} www.karger.com
Yong Xu

Department of Urology, Second Hospital, Tianjin Medical University

23 Pingjiang Road, Hexi District

Tianjin 300211 (China)

Tel +86 228832 8607, Fax +8622 2827 3211, E-Mail liuranlu1976@126.com 


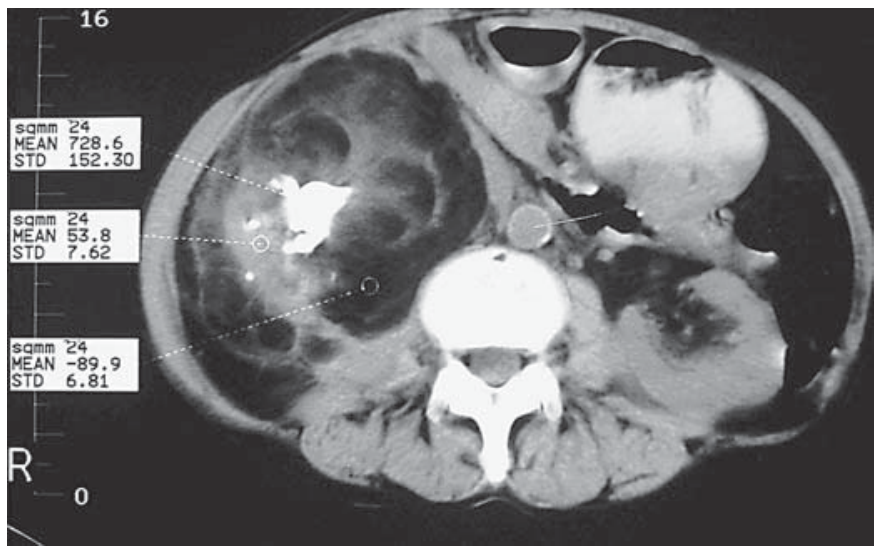

Fig. 1. Transverse contrast-material-enhanced CT scan revealed a mainly low-density and roundish mass with an irregular parenchyma, high-density calcification, and abundant low-density fat.

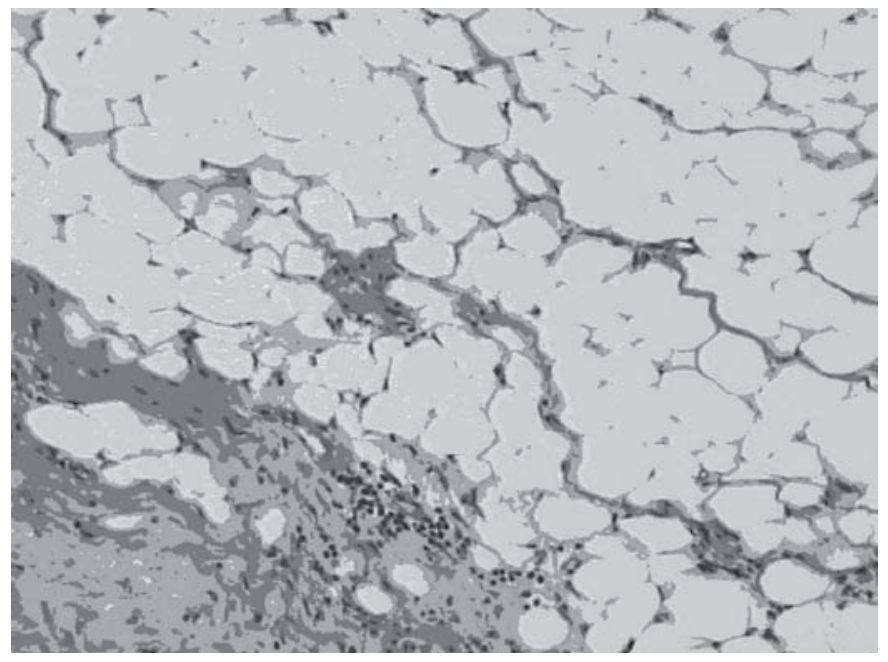

Fig. 3. The hyperplastic fatty tissue at renal sinus, hilum, and periphery consisted of mature fat cells with infiltration of inflammatory cells. HE. $\times 100$.

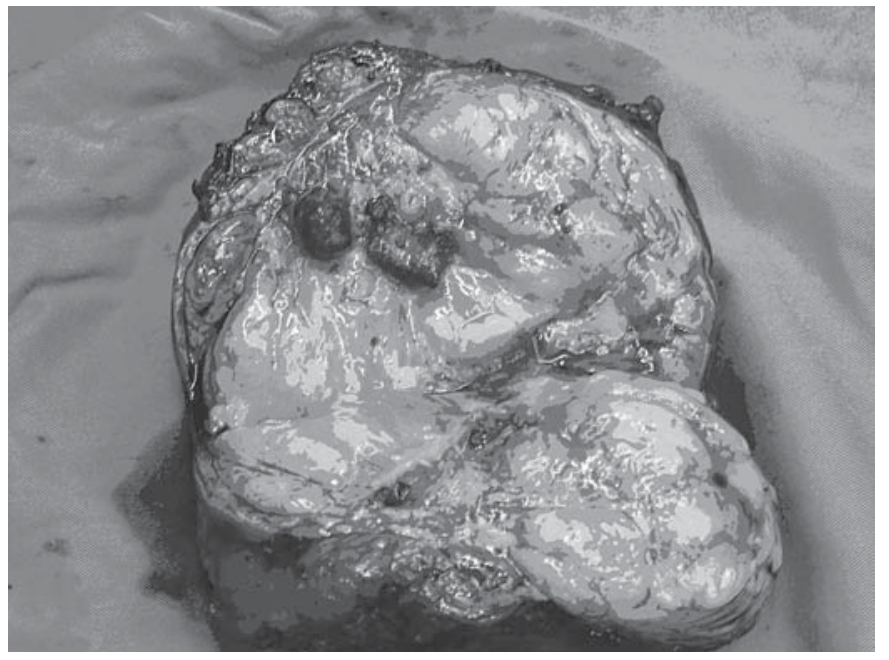

Fig. 2. Macroscopically, a $20 \times 15 \times 10$-cm solid mass, multiple calculi with extremely atrophied renal parenchyma surrounded by overgrown renal sinus, and perirenal fat were present. The whole kidney was almost replaced by fatty tissue.

\section{Discussion}

Replacement lipomatosis of the kidney, also known as replacement fibrolipomatosis, is an advanced form of renal sinus lipomatosis that usually occurs unilaterally. A varying amount of fat and fibrous tissue is always present within the renal sinus which becomes more prominent with aging, obesity, and use of exogenous steroids. Re-

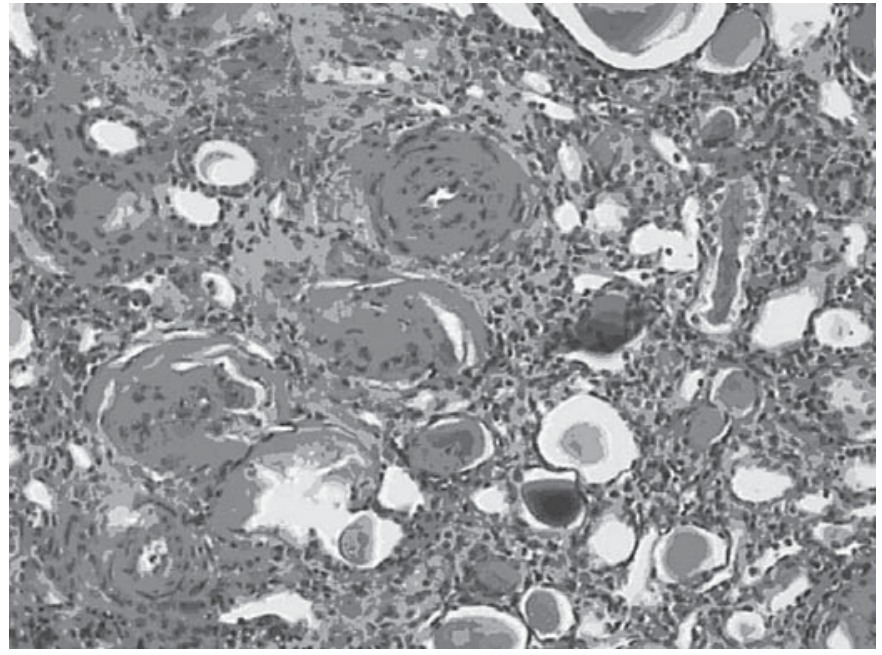

Fig. 4. The renal structure was completely destroyed, and all glomeruli were hyaline with infiltration of a large amount of inflammatory cells. HE. $\times 100$.

placement fibrolipomatosis represents the extreme form of renal sinus lipomatosis, in which infection, long-term hydronephrosis, and calculi are associated with severe renal parenchymal atrophy $[1,2]$. Renal calculus disease associated with inflammatory changes is found in more than $70 \%$ of the cases [3]. Fourteen cases have been reported in China until now; 10 patients (71.4\%) had renal stones. Clinical symptoms, including urinary tract infec- 
tions, fever, and flank pain, usually result from the associated inflammatory and calculus disease [4].

Pathologically, the kidney is usually enlarged and presents with a gross fibrofatty appearance. The renal cortex is extremely atrophied, with varying degrees of hydronephrosis or pyonephrosis as well as acute and chronic pyelonephritic changes. The shape of the kidney is always maintained. There is marked proliferation of fat in the renal sinus, with extremely large fat cells that do not permeate the renal parenchyma but merely develop adjacent to it, as it atrophies $[3,4]$. This process is distinct from that seen with lipomas which are found within the parenchyma.

Abdominal radiographs characteristically demonstrate a staghorn calculus or multiple calculi with an enlarged renal outline. Excretory urographies demonstrate a poorly functioning or nonfunctioning kidney. On ultrasonography (US), there was expansion of the hyperechoic mass that represented lipomatous tissue. A central high-intensity echo with acoustic shadowing represented the staghorn or other calculus. It may be difficult to see the hypoechoic rim of the residual parenchyma [3]. CT is the most accurate method of demonstrating the distinctive features of renal replacement lipomatosis. The calculi and the atrophied renal parenchyma were depicted easily. The abundant fatty tissue centrally has the characteristic attenuation of fat [5]. The characteristic distribution of fat within renal sinus and perinephric space is unique to renal replacement lipomatosis. Magnetic resonance imaging can show three-dimensional images, but, from our point of view, it has no advantage over CT for the diagnosis of renal replacement lipomatosis.

A major differential diagnosis in the presence of longstanding inflammation and calculus obstruction is xanthogranulomatous pyelonephritis (XGP). XGP is a chronic form of infection characterized by destruction of the renal parenchyma. Venous obstruction and hematomas caused by chronic inflammation lead to local accumulation of fat. Histiocytes and englobing fat and forming foam cells are called xanthoma cells. Histologically, the characteristic of XGP is an increase of lipid-laden macrophages (xanthoma cells) in the renal parenchyma, whereas replacement lipomatosis contains large fat cells outside of the renal parenchyma. The features on US and CT are, therefore, different. At US a XGP shows hypoechoic areas that represent purulent material as well as medium-amplitude echoes that correspond to the fibrofatty and/or necrotic debris [3]. A CT shows hydronephrosis or pyonephrosis along with xanthogranulomatous tissue which typically has attenuation values close to those of water.

Additionally, though very rare, XGP and replacement fibrolipomatosis may coexist [6-9]. On the other hand, other focal fatty lesions such as lipomas, angiomyolipomas, and liposarcomas must be considered. The absence of parenchymal atrophy and staghorn calculi are additional clues for excluding these entities [4].

Finally, we want to mention that due to lack of experience with renal replacement lipomatosis, no doctor mentioned this disease during the discussion, instead most doctors focused on a solid mass and tumors, and the other probable diagnosis - XGP - was also ignored. Considering the longtime stone stimulation and her long disease history, some doctors mentioned the possibility of SCC of the pelvis, although the CT findings were not compatible with SCC, thus the case was misdiagnosed before the operation. The diagnosis was difficult to establish, because we had no experience with this disease; the misdiagnosis caused unnecessary psychic pain and burden to the patient and her family, so we hope that more urologists will recognize this disease and avoid learning the same lesson we have learnt.

\section{References}

1 Ambos MA, Bosniak MA, Gordon R, Madayag MA: Replacement lipomatosis of the kidney. AJR Am J Roentgenol 1978;130: 1087-1091.

2 Hurwitz RS, Benjamin JA, Cooper JF: Excessive proliferation of peripelvic fat of the kidney. Urology 1978;11:448-456.

- 3 Subramanyam BR, Bosniak MA, Horii SC, Megibow AJ, Balthazar EJ: Replacement lipomatosis of the kidney: diagnosis by computed tomography and sonography. Radiology 1983;148:791-792.

-4 Karasick S, Wechsler RJ: Case 23: replacement lipomatosis of the kidney. Radiology 2000;215:754-756.

5 Kullendorff B, Nyman U, Aspelin P: Computed tomography in renal replacement lipomatosis. Acta Radiol 1987;28:447-450.

-6 Acunas B, Acunas G, Rozanes I, Buyukbabani N, Gokmen E: Coexistent xanthogranulomatous pyelonephritis and massive replacement lipomatosis of the kidney: CT diagnosis. Urol Radiol 1990;12:88-90.

7 Prasad KK, Pandey R, Kathuria M, Pradhan PK: Co-existent massive renal replacement lipomatosis and xanthogranulomatous pyelonephritis - a case report. Indian J Pathol Microbiol 2003;46:674-675.

8 Sakata Y, Kinoshita N, Kato H, Yamada Y, Sugimura Y: Coexistence of renal replacement lipomatosis with xanthogranulomatous pyelonephritis. Int J Urol 2004;11:44-46.

-9 Kiris A, Kocakoc E, Poyraz AK, Dagli F, Boztosun Y: Xanthogranulomatous pyelonephritis with nephrocutaneous fistula and coexisting renal replacement lipomatosis: the report of a rare case. Clin Imaging 2005;29: 356-358. 